\title{
Diagnóstico tardío y enfermedad avanzada de VIH en pacientes adultos en un hospital de la seguridad social de Perú
}

\author{
Julio Maquera-Afaray, Aleksandar Cvetkovic-Vega, Matlin M. Cárdenas, \\ Helga Kälviäinen y Christian R. Mejia
}

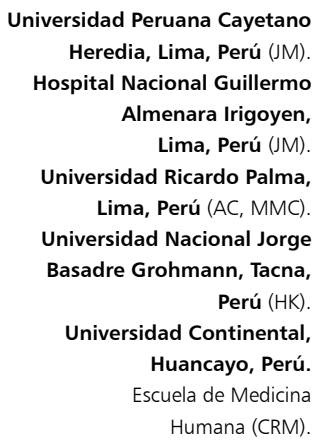

Universidad Peruana Cayetano Heredia, Lima, Perú (JM). Hospital Nacional Guillermo Almenara Irigoyen, Lima, Perú (JM). Universidad Ricardo Palma, Lima, Perú (AC, MMC). Universidad Nacional Jorge Basadre Grohmann, Tacna Perú (HK)

Universidad Continenta Huancayo, Perú Escuela de Medicina Humana (CRM)

Establecimiento donde se realizó el trabajo: Hospital Nacional Guillermo Almenara Irigoyen

Los autores declaran no tener conflictos de interés con la publicación de este artículo. Trabajo auto-financiado.

Recibido: 3 de septiembre de 2015 Aceptado: 10 de julio de 2016

Correspondencia a: Christian R. Mejia christian.mejia.md@gmail.com

\section{Introducción}

L a pandemia de infección por el virus de inmunodeficiencia humana (VIH) comenzó a inicios de los años 80 del siglo pasado. Desde entonces se calcula que aproximadamente más de 78 millones de personas se infectaron y 39 millones fallecieron a causa de enfermedades relacionadas al sindrome de inmunodeficiencia adquirida (SIDA) ${ }^{1}$. La epidemiología global de la infección por VIH ha cambiado debido a la expansión del acceso a la terapia anti-retroviral (TARV): si bien ha aumentado la prevalencia de la infección por $\mathrm{VIH}$, han disminuido su incidencia global y muertes relacionadas $^{2,3}$. En el Perú, a fines de 2014 se han notificado 56.031 casos de infección por VIH y 32.594 casos de SIDA, concentrados principalmente en poblaciones de hombres que tienen sexo con otros hombres (HSH) y mujeres transgénero ${ }^{4,5}$.

En mayo de 2014 se desarrolló el Primer Foro Latinoamericano y del Caribe sobre el continuo de atención de la infección por VIH, abordando el tema "Del diagnóstico al tratamiento efectivo: optimizando las etapas en el continuo de atención". Dentro de las metas programáticas para el año 2020 resalta la de haber efectuado el diagnóstico de $90 \%$ de las personas con infección por VIH; además, se propuso reducir el diagnóstico tardío a menos de 10\%, en personas con diagnóstico reciente. No obstante, en el año 2012, en la mitad de los países, $40 \%$ o más de los pacientes se encontraban en un estadio de enfermedad avanzada (recuento de linfocitos T CD4 $<200$ céls $/ \mathrm{mm}^{3}$ ) en su primera determinación de LT CD4 ${ }^{6,7}$.

El recuento bajo de LTCD4 en el momento diagnóstico es un marcador perjudicial en la infección por VIH, asociado con mayor morbilidad y mortalidad, peor pronóstico y menor supervivencia; asimismo, aquellas personas con infección por VIH/SIDA son menos beneficiadas por la TARV, repercutiendo también de forma importante sobre la utilización y gasto sanitario, y desde la perspectiva de salud pública, aumentaría el riesgo de transmisión del $\mathrm{VIH}^{8-10}$. Investigaciones encuentran asociación entre tener un diagnóstico tardío y variables como el sexo, edad, consumo de drogas y orientación sexual ${ }^{8,11,12}$. En nuestro país, a pesar de ser ésta una enfermedad con altos costos en salud y consecuencias económicas ${ }^{13,14}$, no se encuentran muchos estudios respecto al tema.

El objetivo del estudio fue determinar la frecuencia $\mathrm{y}$ factores asociados al diagnóstico tardío y enfermedad avanzada de la infección por VIH (SIDA) en pacientes atendidos en un hospital referencial y alta complejidad de la seguridad social de Perú. 


\section{Material y Métodos}

\section{Diseño del estudio}

Se realizó un estudio transversal analítico, mediante la revisión de datos secundarios del registro de pacientes con infección por VIH atendidos en el Hospital Nacional Guillermo Almenara Irigoyen (HNGAI) de la seguridad social, Lima-Perú. El seguro social (EsSalud) ofrece servicios de salud a la población asalariada (trabajadores del sector formal y jubilados) y sus familias en sus propias instalaciones; en el año 2012, la población asegurada representó $32,5 \%$ del total de la población estimada del país ${ }^{15}$.

\section{Población y muestra}

Se incluyó a aquellos pacientes adultos con diagnóstico de infección por VIH atendidos en el período comprendido entre 1999 y 2012. Esto es debido a que en 1999 fue aprobado el primer protocolo de TARV del adulto en la seguridad social en Perú ${ }^{16}$. Se excluyó los reportes que no tenían datos coherentes o incompletos requeridos en el presente estudio (exclusión de menos de 3\% de los registros). El muestreo fue de tipo censal, y se empleó como unidad de análisis el registro electrónico de la historia clínica de cada paciente.

\section{Variables}

Basados en las definiciones propuestas por el grupo europeo (European Late Presenter Consensus Working Group) se consideró los análisis correspondientes al primer recuento de $\mathrm{LT} \mathrm{CD} 4+$, realizados en el momento del diagnóstico de infección por $\mathrm{VIH}$, definiendo como enfermedad avanzada (EA) a los que tuvieron un recuento de LT CD4+ $<200$ céls $/ \mathrm{mm}^{3}$ y los que tuvieron un diagnóstico tardio (DT) a los que tuvieron un recuento de LT CD4 $+<350$ céls $/ \mathrm{mm}^{3}$. Adicionalmente fueron analizados datos generales como: edad, sexo, orientación sexual (heterosexual, -HSH- y bisexual) y usuarios/consumidores de drogas ilícitas, como cocaína.

\section{Procedimientos y análisis de datos}

La base de datos primaria fue analizada inicialmente para hacer la depuración, ordenamiento y clasificación de los datos recogidos, según los criterios de inclusión y exclusión. Posteriormente, los datos fueron procesados en el paquete estadístico Stata 11,1® (Stata Corp. Texas, US). Para la estadística descriptiva las variables cuantitativas fueron representadas por medidas de tendencia central y de dispersión, previa evaluación de la distribución de los valores. Las variables cualitativas se analizaron usando frecuencias y porcentajes.

Para la estadística analítica se trabajó con $95 \%$ de confianza; el análisis bivariado se realizó utilizando regresión con Modelos Lineales Generalizados (GLM por sus siglas en inglés), calculando las razones de prevalencia (RP), sus intervalos de confianza al $95 \%$ (IC95\%) y los valores $\mathrm{p}$, con familia Poisson, función de enlace $\log$ y usando modelos robustos para ajustar los resultados. Se trabajó con un nivel de significancia estadística de $\mathrm{p}<0,05$.

\section{Ética de la investigación}

Debido a que fue una investigación realizada a través de la recolección de datos conocidos, el riesgo de investigación fue menor al mínimo. A pesar de esto, se realizó el proceso de aprobación por un Comité de Ética local. Esto sirvió para tener la certeza de que la investigación seguía los parámetros metodológicos y éticos que se norman.

\section{Resulltados}

De los 1.714 datos de pacientes, $71,4 \%$ (1.224) fueron varones y con una mediana de edad de 35 años (rango intercuartílico: 28-42 años de edad). La mayoría refirió ser de orientación sexual heterosexual $(63,5 \%)$, seguidos por HSH (26,3\%) y 6,9\% (153) consumía algún tipo de droga ilícita. El 82,6\% (1.416) fue considerado de DT y $64,5 \%$ (1.106) con una EA. Características adicionales se describen en la Tabla 1.

En la Figura 1 se muestran el gráfico de las edades según el sexo y la presencia de diagnóstico tardío o enfermedad avanzada de infección por VIH.

Al determinar los factores asociados al DT de infección por VIH, el sexo masculino tuvo 17\% más frecuencia de DT comparado con las mujeres (RP:1,17; IC95\%:1,10$1,24 ; \mathrm{p}<0,001)$.

Comparados con el grupo de menos de 40 años de edad, los pacientes entre 41 y 60 años tuvieron $9 \%$ mayor frecuencia de DT (RP: 1,09; IC95\%: 1,04-1,14; $<<0,001$ ) y los que tuvieron más de 60 años tuvieron $14 \%$ mayor frecuencia de DT (RP: 1,14; IC95\%: 1,06-1,22; p <0,001).

Según su orientación sexual, los heterosexuales tuvieron $12 \%$ menor frecuencia de DT comparado con los no heterosexuales (RP: 0,88; IC95\%: 0,84-0,91; $p<0,001$ ), los $\mathrm{HSH}$ tuvieron $8 \%$ mayor frecuencia de DT comparado con los que no fueron HSH (RP: 1,08; IC95\%: 1,04$1,13 ; \mathrm{p}<0,001)$ y los bisexuales tuvieron $18 \%$ mayor frecuencia de DT comparado con quienes no se definían como bisexuales (RP: 1,18; IC95\%: 1,14-1,20; $\mathrm{p}<0,001$ ).

Por último, los consumidores de drogas tuvieron $24 \%$ mayor frecuencia de DT comparado con los que no consumían drogas (RP: 1,24; IC95\%: 1,21-1,27; p < 0,001). (Tabla 2).

Según los factores al diagnóstico de EA de infección por VIH, el sexo masculino tuvo $28 \%$ mayor frecuencia de diagnóstico de EA comparados con las mujeres (RP: 1,28; IC95\%: 1,17-1,40; $\mathrm{p}<0,001)$. 


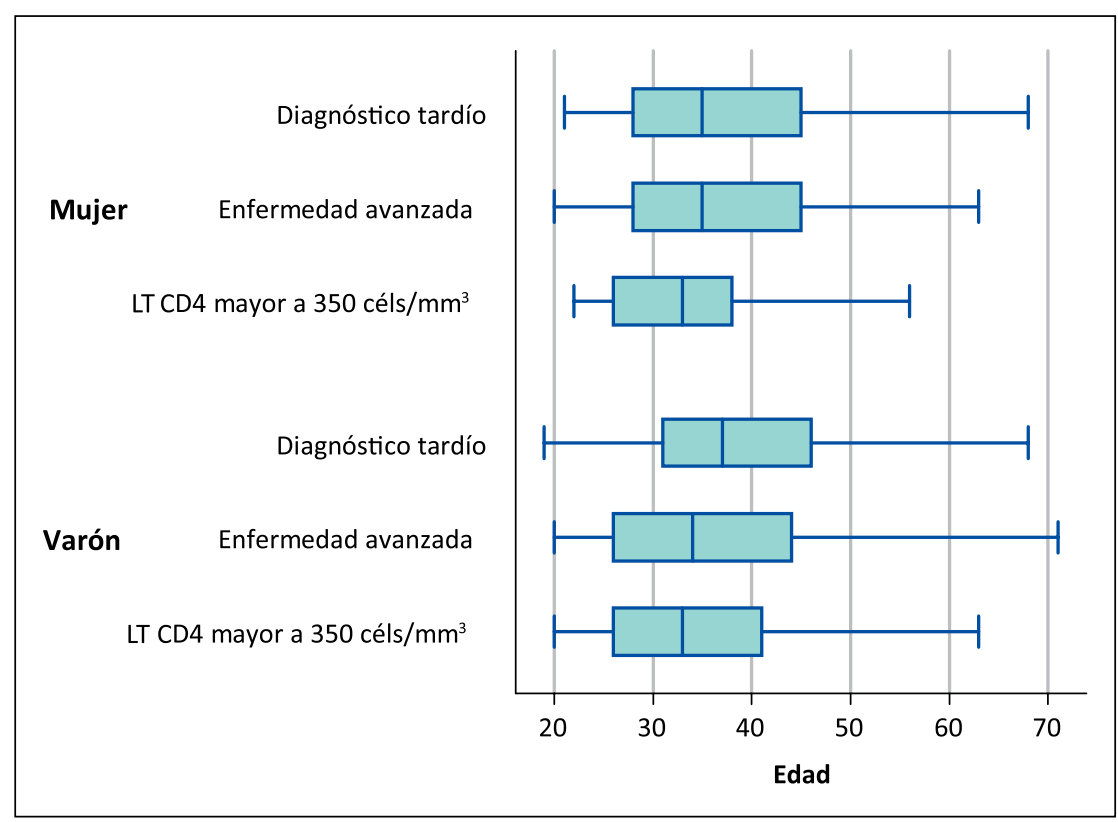

Figura 1. Distribuciones de la edad según el sexo y el tener diagnóstico tardío o enfermedad avanzada.

\begin{tabular}{|c|c|c|}
\hline Variable & $\mathbf{n}$ & $\%$ \\
\hline \multicolumn{3}{|l|}{ Sexo } \\
\hline Masculino & 1.224 & 71,4 \\
\hline Femenino & 490 & 28,6 \\
\hline Edad (años)* & 35 & $28-42$ \\
\hline \multicolumn{3}{|l|}{ Orientación sexual } \\
\hline Heterosexual & 777 & 63,5 \\
\hline Homosexual & 322 & 26,3 \\
\hline Bisexual & 125 & 10,2 \\
\hline Usuarios de drogas & 153 & 6,9 \\
\hline
\end{tabular}

\begin{tabular}{|c|c|c|c|c|c|}
\hline \multirow[t]{2}{*}{ Variable } & \multicolumn{2}{|c|}{ Diagnóstico tardío n (\%) } & \multirow[t]{2}{*}{ Valor $p$} & \multirow[t]{2}{*}{ RP } & \multirow[t]{2}{*}{ IC $95 \%$} \\
\hline & Sí & No & & & \\
\hline Sexo masculino & $1.055(74,5)$ & $169(56,7)$ & $<0,001$ & 1,17 & $1,10-1,24$ \\
\hline \multicolumn{6}{|l|}{ Edad } \\
\hline$<40$ años & $908(64,2)$ & $226(75,8)$ & \multicolumn{3}{|c|}{ Categoría de comparación } \\
\hline 41-60 años & $424(29,9)$ & $64(21,5)$ & $<0,001$ & 1,09 & $1,04-1,14$ \\
\hline$>60$ años & $84(5,9)$ & $8 \quad(2,7)$ & $<0,001$ & 1,14 & $1,06-1,22$ \\
\hline \multicolumn{6}{|l|}{ Orientación sexual } \\
\hline Heterosexual & $637(60,4)$ & $140(82,4)$ & $<0,001$ & 0,88 & $0,84-0,91$ \\
\hline $\mathrm{HSH}$ & $294(27,9)$ & $28(16,6)$ & $<0,001$ & 1,08 & $1,04-1,13$ \\
\hline Bisexual & $124(11,8)$ & $1(0,6)$ & $<0,001$ & 1,18 & $1,14-1,20$ \\
\hline Usuarios de drogas & $153(10,8)$ & $0 \quad(0,0)$ & $<0,001$ & 1,24 & $1,21-1,27$ \\
\hline
\end{tabular}

Comparados con el grupo de menos de 40 años de edad, los que tuvieron entre 41-60 años tuvieron 15\% mayor frecuencia de diagnóstico de EA (RP: 1,15; IC95\%: $1,07-1,24 ; \mathrm{p}<0,001)$ y los que tuvieron más de 60 años tuvieron $23 \%$ mayor frecuencia de diagnóstico de EA (RP: 1,23 ; IC95\%: 1,08-1,39; $p=0,002$ ).

Según su orientación sexual, los heterosexuales tuvieron 19\% menor frecuencia de diagnóstico de EA comparados con los no heterosexuales (RP: 0,81; IC95\%: $0,75-0,87 ; \mathrm{p}<0,001)$ y los bisexuales tuvieron $43 \%$ mayor frecuencia de diagnóstico de EA comparado con los no bisexuales (RP: 1,43; IC95\%: 1,35-1,52; $<<0,001$ ).

Por último, los que usaban drogas tuvieron $64 \%$ mayor frecuencia de diagnóstico de EA comparados con los que no usaban drogas (RP: 1,64; IC95\%: 1,57-1,70; $\mathrm{p}<0,001)$ (Tabla 3). 


\begin{tabular}{|c|c|c|c|c|c|}
\hline \multirow[t]{2}{*}{ Variable } & \multicolumn{2}{|c|}{ Diagnóstico avanzado n (\%) } & \multirow[t]{2}{*}{ Valor $p$} & \multirow[t]{2}{*}{$\mathbf{R P}$} & \multirow[t]{2}{*}{ IC $95 \%$} \\
\hline & Sí & No & & & \\
\hline Sexo masculino & $842(76,1)$ & $382(62,8)$ & $<0,001$ & 1,28 & $1,17-1,40$ \\
\hline \multicolumn{6}{|l|}{ Edad } \\
\hline$<40$ años & $693(62,7)$ & $441(72,5)$ & \multicolumn{3}{|c|}{ Categoría de comparación } \\
\hline 41-60 años & $344(31,1)$ & $144(23,7)$ & $<0,001$ & 1,15 & $1,07-1,24$ \\
\hline$>60$ años & $69(6,2)$ & $23(3,8)$ & 0,002 & 1,23 & $1,08-1,39$ \\
\hline \multicolumn{6}{|l|}{ Orientación sexual } \\
\hline Heterosexual & $492(58,4)$ & $285(74,6)$ & $<0,001$ & 0,81 & $0,75-0,87$ \\
\hline $\mathrm{HSH}$ & $232(27,6)$ & $90(23,6)$ & 0,129 & 1,07 & $0,98-1,16$ \\
\hline Bisexual & $118(14,0)$ & $7 \quad(1,8)$ & $<0,001$ & 1,43 & $1,35-1,52$ \\
\hline Usuarios de drogas & $153(13,8)$ & $0 \quad(0,0)$ & $<0,001$ & 1,64 & $1,57-1,70$ \\
\hline
\end{tabular}

\section{Discusión}

En los últimos años continúan intensificándose esfuerzos globales en la prevención de la transmisión del VIH, habiéndose planteado numerosas iniciativas, como promover el diagnóstico temprano fomentando el testeo para el despistaje de $\mathrm{VIH}^{17}$, con la finalidad de minimizar la problemática del diagnóstico tardío y enfermedad avanzada.

Tener un diagnóstico tardío genera un pobre pronóstico, que lleva a una mala calidad de vida en lo que respecta a la salud y al rol del paciente en la sociedad ${ }^{18,19}$. Estudios realizados sobre diagnóstico tardío han demostrado porcentajes cercanos a $30 \%$, principalmente en países de altos ingresos ${ }^{20-24}$, en contraste con lo reportado en países de bajos y medianos ingresos, con porcentajes de $77 \%$, $78 \%$ y 55\%, para África, Asia y Latinoamérica, respectivamente ${ }^{25}$; Sin embargo, los reportes latinoamericanos varían entre sí, respecto la prevalencia de DT en la región, habiéndose encontrado 44\% en Brasil ${ }^{26}$ y $61 \%$ en Méxi$\mathrm{co}^{27}$. Aún más, Crabtree-Ramírez y cols., demostraron que alrededor de $76 \%$ de los pacientes iniciaban tardíamente la TARV en seis países de Latinoamérica y el Caribe (56\% Argentina, 80\% Chile, 76\% Haití, 91\% Honduras, $79 \%$ México y $86 \%$ Perú), de los cuales $55 \%$ tuvo un testeo tardío ${ }^{27}$. Nuestros resultados muestran porcentajes superiores tanto de DT $(82,6 \%)$, como de EA (64,5\%), constituyendo un problema relevante de salud pública en Perú, debido que los pacientes con estas condiciones acudirían a recibir atención médica, en su mayoría, con un deterioro clínico e inmunológico que no sólo repercutiría sobre la salud del individuo, sino también del colectivo, y del propio sistema de salud ${ }^{28-30}$.

En el análisis se encontró que los pacientes de sexo masculino y que los grupos sobre de 40 años de edad tienen mayor frecuencia de DT; estos datos se ven respaldados por hallazgos en estudios que indican que tanto la edad como el sexo se asocian a un DT de infección por $\mathrm{VIH}^{31,32}$. Smith y cols., encontraron una mayor frecuencia de DT y mortalidad en población adulta mayor con infección por $\mathrm{VIH}^{32}$, pudiéndose explicar por ciertos factores sociales y de hábitos de riesgo; también la inmunosenescencia propia del envejecimiento, reduce su potencial para recuperarse de la infección por $\mathrm{VIH}^{31-33}$. Además, la orientación sexual HSH y bisexual tuvieron más frecuencia de DT. Blas y cols., identificaron en población peruana de HSH que " $e l$ miedo de tener un resultado positivo y el desconocimiento de lugares donde realizarse la prueba de VIH' son las razones más comunes para no realizarse la prueba de ELISA para $\mathrm{VIH}^{33}$, otras razones reconocidas son el estigma de tener infección por $\mathrm{VIH}$, el nivel de conocimiento y/o la amenaza contra la identidad personal ${ }^{29}$. Los usuarios de drogas también tuvieron mayor DT; esto guarda relación con algunos estudios que demuestran que los usuarios de drogas ilícitas tienen mayor probabilidad de tener DT e inclusive $\mathrm{EA}^{35-37}$.

Por otro lado, en más de la mitad de los pacientes fue diagnosticada la infección por VIH como EA, denotando que la prueba de VIH se realiza principalmente cuando existe un deterioro evidente de la salud ${ }^{29,30}$. La mayor frecuencia de EA estuvo en el sexo masculino, la orientación sexual-heterosexual y bisexual-, y consumo 
de drogas ilícitas, similar al reportado por múltiples investigaciones $^{38,39}$. Encontramos que los heterosexuales tuvieron menores frecuencias de diagnóstico tardío y enfermedad avanzada; esto contradice a lo que refiere la literatura médica encontrada, ya que ésta menciona que los heterosexuales generalmente son un grupo que suele tener presentaciones tardías de esta enfermedad ${ }^{19,35,37,38,40}$; esto se puede deber a que actualmente los HSH suelen tener una mayor percepción de riesgo del contagio y los síntomas de la enfermedad, en comparación a los otros grupos, pudiendo acudir oportunamente a la realización de la prueba de ELISA para VIH. Sin embargo, en nuestra revisión, encontramos que tanto la población HSH como bisexuales tuvieron mayor frecuencia de DT y EA.

La vigente norma técnica de salud de atención integral del adulto con infección por VIH en Perú ${ }^{40}$, establece que la prueba de tamizaje es voluntaria y previa consejería, y es ofertada a toda persona con diagnóstico de tuberculosis pulmonar o extra-pulmonar, hepatitis B, sífilis y otras infecciones de transmisión sexual. Sin embargo, consideramos esta condición como una barrera para el testeo oportuno, ya que la falta de percepción de riesgo, de tener la infección por VIH, en la población es una de las principales razones por la que no se realizan la prueba voluntariamente ${ }^{41}$.

Como las principales limitaciones del estudio reportamos el sesgo de información y de selección; la primera debido a que la información fue obtenida mediante registros electrónicos de las historias clínicas, por lo que tampoco se pudo obtener más variables, que hubiesen sido importantes para caracterizar a la población estudiada. El segundo sesgo se generó por no usar un muestreo que nos permita inferir a toda la población, siendo estudiado sólo un grupo atendido en un solo centro hospitalario. A pesar de esto, los resultados son importantes, ya que muestran asociaciones compatibles con estudios realizados y en una gran cantidad de pacientes diagnosticados en diferentes años, siendo relevantes debido a la escasez de información en Perú, lo que puede tomarse como resultados iniciales para generar más investigación en el tema. Además, en el presente estudio no evaluamos a los presentadores tardíos, definidos como la demora entre la primera prueba positiva de infección por VIH y la presentación para recibir cuidado/manejo de la enfermedad.

Concluimos que, es alta la frecuencia de DT y EA en la población estudiada; además, los factores asociados a una mayor frecuencia de DT y EA, fueron el ser de sexo masculino, pertenecer al grupo etario sobre 40 años de edad, orientación sexual (HSH y bisexual) y el consumir drogas.
Nuestros resultados reflejan la persistencia de barreras que limitan la efectividad de la respuesta en la lucha contra el VIH en Perú ${ }^{43}$. Por tanto, es necesario fortalecer la articulación entre los sub-sistemas del sector salud peruano (Ministerio de Salud, Seguridad Social, Sanidades de las Fuerzas Armadas y Policiales, y sector privado) que permita estandarizar las intervenciones, reforzando las políticas de prevención y promoción en la infección por VIH, permitiendo mayor acceso a las pruebas diagnósticas en la población, no sólo como una prueba de tamizaje voluntario sino como una prueba de rutina en la atención médica, enfatizando en aquellos grupos de riesgo; y concientizando a los profesionales de la salud sobre la importancia de la identificación temprana de la infección por VIH, evitando perder la oportunidad de solicitar la prueba que diagnostique o descarte la infección.

Agradecimientos. A la Asociación Médica de Investigación y Servicios en Salud (AMISS) y a la Sociedad Científica de Estudiantes de Medicina de la Universidad Ricardo Palma (SOCEMURP) por la capacitación constante durante el análisis de datos y desarrollo del presente trabajo.

\section{Resumen}

Introducción: La demora en el diagnóstico de la infección por VIH mediante el recuento de LT CD4 es un problema de salud pública. Objetivo: Determinar la frecuencia y factores asociados al diagnóstico tardío (DT) y enfermedad avanzada (EA) de infección por $\mathrm{VIH}$ en pacientes atendidos en un hospital peruano. Materiales y Métodos: Estudio transversal analítico de datos secundarios de pacientes adultos con diagnóstico de infección por VIH atendidos durante el período 1999-2012. Resultados y Discusión: De 1.714 pacientes, $82,6 \%$ (1.416) tuvo DT y 64,5\% (1.106) EA. Estuvieron asociados con una mayor frecuencia: el sexo masculino (DT: $17 \%$ y EA: 28\%; p < 0,001), edad entre 41-60 años (DT: 9\% y EA: 15\%; p < 0,001), edad mayor a 60 años (DT: 14\% y EA: 23\%; p <0,003), orientación bisexual (DT: 18\% y EA: 43\%; $p<0,001$ ), orientación homosexual (DT: $8 \%$; $<<0,001$ ) y usuarios de drogas (DT: $24 \%$ y EA: 64\%; p < 0,001). El ser heterosexual estuvo asociado a una menor frecuencia (DT: $12 \%$ y EA: $19 \% ; \mathrm{p}<0,001)$. Conclusión: Se encontró una alta la frecuencia de DT y la EA, y los factores asociados a éstas fueron: sexo masculino, grupos sobre 40 años de edad, grupos sexuales de riesgo (homosexuales y bisexuales) y consumidores de drogas. 


\section{Referencias bibliográficas}

1.- ONU SIDA. Hoja Informativa 2014. Datos Estadísticos mundiales 2014. [Acceso en agosto de 2015]. Disponible en: http://www.unaids.org/sites/default/files/ media_asset/20140716_FactSheet_es_0.pdf

2.- Maartens G, Celum C, Lewin S R. HIV infection: epidemiology, pathogenesis, treatment, and prevention. Lancet 2014; 384 (9939): 258-71.

3.- Joint United Nations Programme on HIV/AIDS (UNAIDS). Global report: UNAIDS report on the global AIDS epidemic 2013 [Acceso en agosto de 2015]. Disponible en: http://www. unaids.org/sites/default/files/media_asset/ UNAIDS_Global_Report_2013_en_1.pdf

4.- Dirección General de Epidemiología Ministerio de Salud del Perú. Boletín Epidemiológico Mensual de VIH/SIDA. 2014 [Acceso en julio de 2015]. Disponible en: http://www.dge.gob. pe/portal/docs/vigilancia/vih/Boletin_2014/ diciembre.pdf

5.- Reyes M F, Pun Chinarro M. Análisis de la situación de la epidemia del VIH SIDA Perú 2013. Disponible en: http://www.dge.gob.pe/ portal/docs/ASISVIH2013.pdf [Acceso en julio de 2015].

6.- Organización Panamericana de la Salud. Tratamiento antirretroviral bajo la lupa: un análisis de salud pública en Latinoamérica y el Caribe 2013. Disponible en: http:// www.paho.org/hq/index.php?option $=\mathrm{com}_{-}$ docman\&task $=$ doc_view\&gid $=23711 \&$ Itemid $=$ [Acceso en junio de 2015].

7.- Organización Panamericana de la Salud. Tratamiento antirretroviral bajo la lupa: un análisis de salud pública de Latinoamérica y el Caribe 2014. Disponible en: http://www.paho. org/hq/index.php?option=com_topics\&view= rdmore $\&$ cid $=7167 \&$ Itemid $=40737 \&$ lang $=$ es [Acceso en agosto de 2015].

8.- Hoffman J, van Griensven J, Colebunders R, McKellar M. Role of the CD4 count in HIV management. HIV Ther 2010; 4 (1): 27-39.

9.- Kozak M, Zinski A, Leeper C, Willig J H, Mugavero M J. Late diagnosis, delayed presentations and late presentation in HIV: proposed definitions, methodological considerations and health implications. Antivir Ther 2013; 18: 17-23.

10.- Fleishman J A, Yehia B R, Moore R D, Gebo K A. The economic burden of late entry into medical care for patients with HIV infection. Med Care 2010; 48 (12): 1071-9.

11.- Yong M K, Elliott J H, Woolley I J, Hoy J F. Low CD4 count is associated with an increased risk of fragility fracture in HIV-infected patients. J Acquir Immune Defic Syndr 2011; 57 (3): 205-10

12.- Adler A, Mounier-Jack S, Coker R J. Late diagnosis of HIV in Europe: definitional and public health challenges. AIDS Care 2009; 21 (3): 284-93

13.- MINSA-Perú. Medición del Gasto en SIDA -MEGAS Informe Final 2011. Disponible en: http://www.unaids.org/sites/default/files/en/ media/unaids/contentassets/documents/dataand-analysis/tools/nasa/NASA_Peru_2010_ es.pdf [Acceso en agosto de 2015].

14.- MINSA-Perú. Informe Nacional Sobre los Progresos Realizados en el País 2013. Disponible en: http://www.unaids.org/sites/ default/files/en/dataanalysis/knowyourresponse/ countryprogressreports/2014countries/PER narrative_report_2014.pdf [Acceso en agosto de 2015].

15.- Seguridad Social (EsSalud). Memoria Institucional Anual 2012. Disponible en: http://www.essalud.gob.pe/transparencia/pdf/ memoria/memoria_2012.pdf [Acceso en agosto de 2015].

16.- Gerencia Central de Prestaciones de Salud (EsSalud). Guía de manejo de terapia antirretroviral para pacientes adultos con infección por VIH/SIDA en EsSalud. 2009 Disponible en: http://apps.who.int/ medicinedocs/documents/s18031es/s18031es. pdf [Acceso en agosto de 2015].

17.- Yazdanpanah Y, Lange J, Gerstoft J, Cairns G. Earlier testing for HIV - how do we prevent late presentation? Antivir Ther 2010; 15 (Suppl 1): $17-24$.

18.- Rodríguez J, Prieto S, Correa C, Forero M F, Pérez C, Soracipa Y, et al. Teoría de conjuntos aplicada al recuento de linfocitos y leucocitos: predicción de linfocitos T CD4 de pacientes con virus de la inmunodeficiencia humana/SIDA. Inmunología 2013; 32 (2): 50-6.

19.- Diez Y M, Exterior S, Sanidad D. Diagnóstico tardío de la infección por VIH: Situación en España. 2011 [Acceso el 16 de agosto de 2015]; Available from: http://msssi.es/ca/ciudadanos/ enfLesiones/enfTransmisibles/sida/vigilancia/ DiagnosticoTardio2011.pdf

20.- Althoff K N, Gange S J, Klein M B, Brooks J T, Hogg R S, Bosch R J, et al. Late presentation for human immunodeficiency virus care in the United States and Canada. Clin Infect Dis 2010; 50 (11): 1512-20.

21.- De Olalla P G, Manzardo C, Sambeat M A, Ocaña I, Knobel H, Humet V, et al. Epidemiological characteristics and predictors of late presentation of HIV infection in Barcelona (Spain) during the period 2001-2009. AIDS Res Ther 2011; 8 (1): 22

22.- Girardi E, Aloisi M S, Arici C, Pezzotti P, Serraino D, Balzano R, et al. Delayed presentation and late testing for HIV: demographic and behavioral risk factors in a multicenter study in Italy. J Acquir Inmune Defic Syndr 2004; 36 (4): 951-9.

22.- Ndiaye B, Salleron J, Vincent A, Bataille P,
Bonnevie F, Choisy P, et al. Factors associated with presentation to care with advanced HIV disease in Brussels and Northern France: 1997-2007. BMC Infect Dis 2011; 11 (1): 11

23.- Oliva J, Díez M, Galindo S, Cevallos C, Izquierdo A, Cereijo J, et al. Predictors of advanced disease and late presentation in new HIV diagnoses reported to the surveillance system in Spain. Gac Sanit 2014; 28 (2): 116-22.

24.- The ART-LINC Collaboration of the International Databases to Evaluate AIDS (IeDEA). Antiretroviral therapy in resource-limited settings 1996 to 2006: patient characteristics, treatment regimens and monitoring in sub-Saharan Africa, Asia and Latin America: ART in resourcelimited settings. Trop Med Int Health 2008; 13 (7): 870-9.

25.- Grangeiro A, Escuder M M L, Pereira J C R. Late entry into HIV care: lessons from Brazil, 2003 to 2006. BMC Infect Dis 2012; 12 (1): 99.

26.- Crabtree-Ramírez B, Caro-Vega Y, Belaunzarán-Zamudio F, Sierra-Madero J. High prevalence of late diagnosis of HIV in Mexico during the HAART era. Salud Publica Mex 2012; 54: 506-14.

27.- Crabtree-Ramírez B, Caro-Vega Y, Shepherd B E, Wehbe F, Cesar C, Cortés C, et al. Cross-sectional analysis of late HAART initiation in Latin America and the Caribbean: Late testers and late presenters. PLoS ONE 6 (5): e20272. doi:10.1371/journal. pone.0020272.

28.- Apodaca M J F-R de, Molero F, Nouvilas E, Arazo P, Dalmau D. Determinants of late diagnosis of HIV infection in Spain. Psychosoc Interv 2014; 23 (3): 177-85.

29.- Goodall L, Leen C. Late diagnosis of HIV: could this be avoided? Scott Med J 2011; 56 (2): 84-6.

30.- Díez M. Diagnóstico tardío de la infección por VIH. Rev Esp Sanid Penit 2011; 13 (2): 35-7.

31.- Lewis J, Walker A S, Castro H, De Rossi A, Gibb , Giaquinto C, et al. Age and CD4 count at initiation of antiretroviral therapy in HIV-infected children: Effects on long-term T-cell reconstitution. J Infect Dis 2012; 205 (4): 548-56.

32.- Smith R D, Delpech V C, Brown A E, Rice B D. HIV transmission and high rates of late diagnoses among adults aged 50 years and over. AIDS 2010, 24: 2109-15.

33.- Blas M M, Alva I E, Cabello R, Carcamo C, Kurth A E. Risk behaviors and reasons for not getting tested for HIV among men who have sex with men: An online survey in Peru. PLoS ONE 6 (11): e27334. doi:10.1371/journal. pone. 0027334

34.- Cevallos García C, Verdejo Ortés J, 
Martínez Rodríguez S, Izarra Pérez C. Late diagnosis of human immunodeficiency virus infection in the Madrid Region (2007-2011). Rev Esp Salud Pública 2012; 86 (1): 37-47.

35.- Wanyenze R K, Kamya M R, Fatch R, Mayanja-Kizza H, Baveewo S, Sawires S, al. Missed opportunities for HIV testing and late-stage diagnosis among HIV-infected patients in Uganda. Myer L, editor. PLoS ONE 2011; 6 (7): e21794.

36.- Martín Suárez I, Cano Monchul R, Pérez de Ayala P, Aguayo Canela M, Cuesta F, Rodríguez P, et al. Calidad de vida, aspectos psicológicos y sociales en pacientes con infección VIH avanzada. In: Anales de Medicina Interna [Internet]. SciELO Espana; 2002 [Acceso el 17 de agosto de 2015]. p. 20-8. Available from: http:// scielo.isciii.es/scielo.php?pid=S0212$71992002000800003 \&$ script $=$ sci arttext

37.- Reus S, Portilla J, Gimeno A, Sánchez-Payá J, García-Henarejos JA, Martínez-Madrid O, et al. Predictores de progresión y muerte en pacientes con infección avanzada por el VIH en la era de los tratamientos anti-retrovirales de gran actividad. Enferm Infecc Microbiol Clin 2004; 22 (3): 142-9.

38.- Tey J S, Ang L W, Tay J, Cutter J L, James L, Chew S K, et al. Determinants of late-stage HIV disease at diagnosis in Singapore, 1996 to 2009. Ann Acad Med Singap 2012; 41 (194): 9.

39.- Mukolo A, Villegas R, Aliyu M, Wallston K A. Predictors of late presentation for HIV diagnosis: a literature review and suggested way forward. AIDS Behav (2013) 17: 5-30.

40.- Ministerio de Salud, Dirección General de Salud de las Personas, Estrategia Sanitaria Nacional de Prevención y Control de Infecciones de Transmisión Sexual y VIH/SIDA. Norma Técnica de Salud de Atención Integral del Adulto con Infección por el Virus de la Inmunodeficiencia Humana (VIH). 2014.

41.- Mills CW, Sabharwal CJ, Udeagu C-C, Bocour A, Bodach S, Shepard C, et al. Barriers to HIV testing among HIV/AIDS concurrently diagnosed persons in New York City. Sex Transm Dis 2011; 38 (8): 715-21.

42.- Ministerio de Salud, Dirección General de Epidemiología. Análisis de la Situación Epidemiológica del VIH/SIDA en el Perú, 2013. 